\title{
Synthesis and Photocrosslinking of Disulfonated Poly(arylene ether sulfone) Copolymers for Potential Reverse Osmosis Membrane Materials
}

\author{
Ali Nebipasagil, ${ }^{a}$ Benjamin J. Sundell, ${ }^{a}$ Ozma R. Lane, ${ }^{a}$ Sue J. Mecham, ${ }^{b^{*}}$ Judy S. Riffle, ${ }^{a}$ and \\ James E. McGrath ${ }^{a}$ \\ ${ }^{a}$ Macromolecules and Interfaces Institute, Virginia Tech, Blacksburg, VA 24061, USA \\ b257 Caudill Labs, Campus Box 3290, University of North Carolina Chapel Hill, Chapel Hill, \\ NC 27599, USA
}

* To whom correspondence should be addressed, sjmecham@email.unc.edu

Keywords: disulfonated poly(arylene ether sulfone); oligomer synthesis; telechelic polyacrylamide; UV crosslinking; thin films

\section{Abstract:}

Disulfonated poly(arylene ether sulfone) random copolymers with controlled oligomeric molecular weights were synthesized via nucleophilic aromatic substitution step polymerization. A monofunctional endcapping reagent, meta-aminophenol, was utilized to control the molecular weight of the oligomers and to install telechelic amine endgroups. The meta-aminophenol endcapped oligomers were reacted with acryloyl chloride to obtain novel crosslinkable poly(arylene ether sulfone) oligomers with acrylamide groups on both ends. The chemical compositions of the functional oligomers were characterized by ${ }^{1} \mathrm{H}$ NMR spectroscopy to determine the degree of sulfonation and concentrations of acrylamide endgroups. The acrylamide-terminated oligomers were crosslinked with UV radiation in the presence of a multifunctional acrylate and a UV photoinitiator. Transparent, free-standing, dense films were obtained with high gel fractions. FTIR spectroscopy was utilized to observe the progress of the photo-crosslinking reaction. Thin films were cast from either aqueous or water-methanol solutions. The crosslinked disulfonated poly(arylene ether sulfone) networks exhibited reduced water uptake and swelling relative to their linear counterparts. SEM and AFM showed that the photo-crosslinked thin films had smooth surfaces.

\section{Introduction}

Radiation initiated polymerization has been widely applied to manufacturing. UV photopolymerization is the most commonly practiced method; applications include thin films, coatings, paints, printer inks, biomedicine, electronics and photoresist nanofabrication. ${ }^{1-6} \mathrm{UV}$ initiated photo-polymerization has been applied to thin film manufacturing due to its rapid production rate, uniform crosslinking and products with low volatile content.

In general, UV curable resins are often solutions of monomers, oligomers and other reagents in a solvent which solidify to form a film through UV initiated crosslinking. A polymer film emerges, when a resin is applied on a surface, as the solvent evaporates and the chemical reactions form crosslinks. As the solvent evaporates, viscosity, density and $\mathrm{T}_{\mathrm{g}}$ increase. Therefore, the rate of 
solvent evaporation becomes dependent on the solvent's diffusion rate to the surface. ${ }^{7}$ If a film is formed at temperatures below the $\mathrm{T}_{\mathrm{g}}$ of the neat polymer it will harden to form a solid polymer layer, but the film retains considerable amounts of solvent which requires further processing steps for full removal. ${ }^{7}$ Waterborne UV curable resins offer some advantages over conventional formulations including reduced toxic volatiles content, ${ }^{7,8}$ and therefore they are widely considered environmentally friendly alternatives to their solvent-based counterparts.

Poly(arylene ether sulfone)s are well-known hydrophobic, amorphous polymers that display excellent thermal and mechanical properties, as well as high chemical resistance against oxidation, chain scission and hydrolysis. ${ }^{9-12}$ The direct copolymerization of sulfonated and nonsulfonated co-monomers to synthesize sulfonated poly(arylene ether sulfone)s avoids any unwanted side reactions or degradation of polymers that often occur during post-sulfonation. Additionally, direct copolymerization provides the advantages of distributing the sulfonate units homogeneously throughout the polymer, as well as gaining control over the degree of sulfonation. This method leads to high molecular weight copolymers and good reproducibility. ${ }^{13,14}$ Water uptake and consequent swelling of these copolymers increase as the level of sulfonation is increased, and at high ion exchange capacities this results in water solubility and loss of mechanical stability. ${ }^{15,16}$ Crosslinking can reduce the swelling of these highly hydrophilic materials and improve their mechanical stability. ${ }^{17} \mathrm{We}$ have previously reported crosslinking of phenoxide-terminated polysulfones with $50 \%$ of the repeat units disulfonated with a tetrafunctional epoxide, and shown that networks with gel fractions up to $85 \%$ could be produced whereas the crosslinks reduced water uptake by $70 \% .^{17}$

Over the past several decades membrane-based separation technologies have been used to augment desalination as a viable source of fresh water. ${ }^{18}$ Reverse osmosis (RO) is a thin membrane-based separation method where saline water is pressurized against a semipermeable membrane. State-of-the-art membranes are thin films with a highly crosslinked aromatic polyamide as the selective layer. Commercial products are manufactured by synthesizing a polyamide interfacially directly onto the surface of a poly(arylene ether sulfone) microporous film, and this results in a thin selective layer of crosslinked polyamide. Poly(arylene ether sulfone) is the primary support material used in state-of-the-art thin film composites. ${ }^{19}$ Given their excellent attributes, these materials are ideal for the membrane applications. They are not a suitable replacement for the selective layer in membrane separations since such applications require some hydrophilicity to produce reasonable levels of water flux. Incorporation of sulfonate moieties into poly(arylene ether sulfone)s introduces hydrophilicity into these hydrophobic polymers, and they are beneficial to other physical properties such as $\mathrm{T}_{\mathrm{g}}{ }^{20,21}$ Therefore sulfonated poly(arylene ether sulfone)s have been considered as potential candidates for the selective layers on RO thin film composites.

Fabrication of thin film composite (TFC) membranes is one approach to preparing water purification membranes utilizing disulfonated poly(arylene ether sulfone)s. ${ }^{22}$ Multiple layers of high molecular weight random poly(arylene ether sulfone) copolymer with either 20 or 32 mole $\%$ of the disulfonated units were cast into thin films from dilute solutions onto a UDEL ${ }^{\circledR}$ support. This yielded a disulfonated polysulfone selective layer, similar to the state-of-the-art commercial thin film membranes. Di(ethylene glycol) was identified as a benign solvent that selectively dissolved the disulfonated poly(arylene ether sulfone) copolymers whereas the UDEL ${ }^{\circledR}$ support 
1

was insoluble. Defect-free films had salt rejections around $97 \%$ with a significant improvement in water flux relative to their thicker dense membrane counterparts. ${ }^{22}$

Our group has reported the synthesis of telechelic disulfonated oligomers with phenol and aromatic amine functionalities crosslinked with epoxy reagents previously. ${ }^{17,23}$ Herein we report the synthesis of acrylamide-terminated disulfonated poly(arylene ether sulfone) oligomers and crosslinking of the new materials with multifunctional acrylate co-monomers using UV light. This study focuses on the synthesis, characterization and fabrication of these photo-curable materials into thin films.

\section{Experimental \\ Materials}

4,4'-(Propane-2,2-diyl)diphenol (bisphenol A) and 4,4'-dichlorodiphenylsulfone (DCDPS, 99\%) were kindly provided by Solvay and recrystallized from toluene before use. 3,3'-Disulfonated4,4'-dichlorodiphenylsulfone (SDCDPS, 98\%) was purchased from Akron Polymer Systems (Akron, OH). ${ }^{24}$ Potassium carbonate $\left(\mathrm{K}_{2} \mathrm{CO}_{3}, 99 \%\right)$, 2-propenoyl chloride (acryloyl chloride, 96\%), triethylamine (TEA, 99.5\%), pentaerythritol tetraacrylate, diethylene glycol diacrylate, glycerol propoxylate triacrylate, trimethylolpropane ethoxylate triacrylate, 2,2-dimethoxy-2-

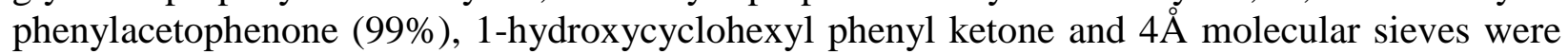
purchased from Sigma Aldrich. Calcium hydride $\left(\mathrm{CaH}_{2}, 90-95 \%\right)$ was purchased from Alfa Aesar. $m$-Aminophenol (99\%) and $N, N$-dimethylacetamide (DMAc, 99\%) were purchased from Acros Organics. DMAc was distilled from calcium hydride and stored over activated molecular sieves before use. HPLC-grade water was purchased from Spectrum Chemicals. Toluene and isopropanol were purchased from Fisher Scientific. The solvents were used as received unless noted otherwise. Table 1 shows the structures and acronyms of the crosslinking reagents and photoinitiators.

Table 1. Names and structures of crosslinking agents and photoinitiators

. 


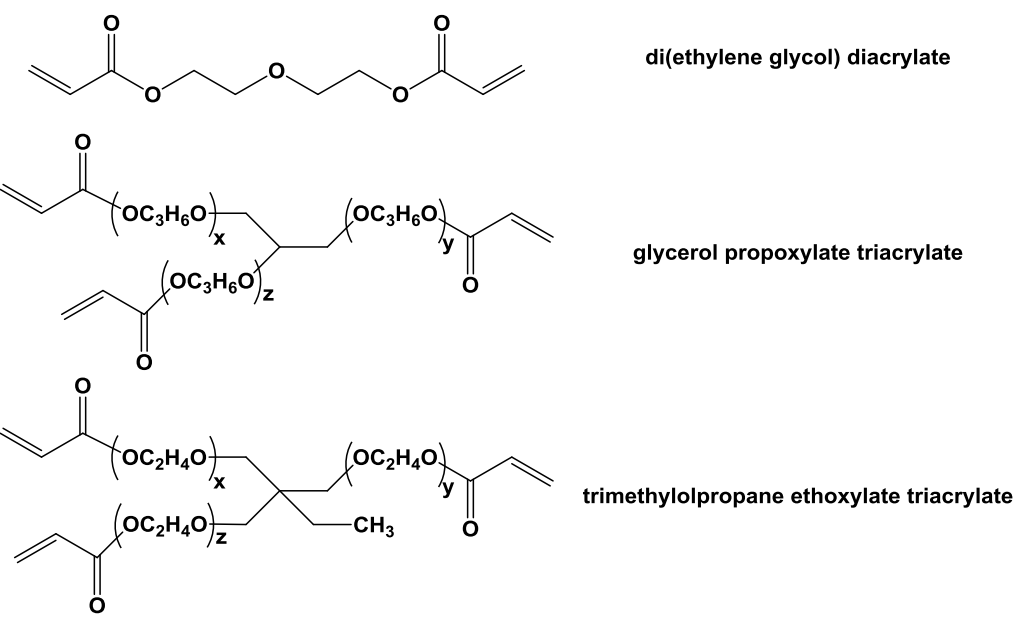<smiles>C=CC(=O)OCC(COC(=O)C=C)(COC(=O)C=C)COC(=O)C=C</smiles>

pentaerythritol tetraacrylate (PETA)<smiles>COC(OC)(C(=O)c1ccccc1)c1ccccc1</smiles>

2,2-dimethoxy-2-phenylacetophenone (DMPA)

1-hydroxycyclohexyl phenyl ketone (HCPK)

Synthesis of a 5,000 $\mathrm{g} / \mathrm{mol}\left(M_{n}\right)$ amine-endcapped disulfonated polysulfone copolymer (AMBisASXX, Figure 1))<smiles>O=C(c1ccccc1)C1(O)CCCCC1</smiles>

Poly(arylene ether sulfone) oligomers with controlled degrees of sulfonation and controlled molecular weights were synthesized via nucleophilic aromatic substitution. SDCDPS was incorporated into the oligomer via direct copolymerization as previously reported. ${ }^{13,14}$

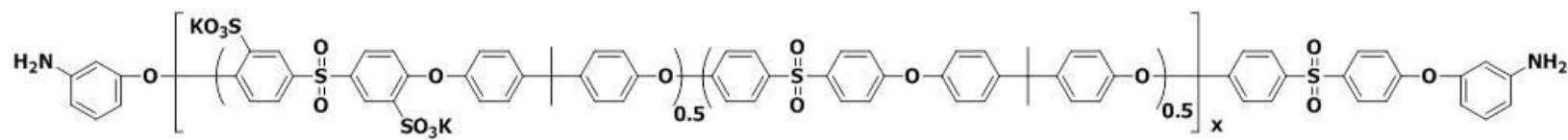

Figure 1. Amine-endcapped disulfonated poly(arylene ether sulfone) with $50 \%$ of the repeat units disulfonated (AM-BisAS50)

High monomer purity and a strict monomer ratio are imperative to obtain high conversion with precisely controlled molecular weights and composition. Bisphenol A and DCDPS were recrystallized from toluene to eliminate impurities. Hygroscopic reagents can absorb moisture under ambient conditions. Thus $\mathrm{K}_{2} \mathrm{CO}_{3}$ was dried for $24 \mathrm{~h}$ and SDCDPS for $72 \mathrm{~h}$ at $160{ }^{\circ} \mathrm{C}$ under vacuum to remove moisture. $\mathrm{NaCl}$ is a by-product of SDCDPS synthesis and remains as an inert 
impurity in SDCDPS. The $\mathrm{NaCl}$ content in the SDCDPS was quantified as $3 \mathrm{~mol} \%$ by UV-VIS spectroscopy before it was used in the oligomer synthesis. ${ }^{25}$ A representative synthetic procedure is provided below. Bisphenol A (43.6 mmol, $9.9534 \mathrm{~g}$ ), DCDPS (25 mmol, 7.1790 g), SDCDPS $(25 \mathrm{mmol}, 12.6611 \mathrm{~g})$, and $\mathrm{m}$-aminophenol (12.7 mmol, $1.3999 \mathrm{~g})$ were weighed into a $250-\mathrm{mL}$ three-necked flask and dissolved in DMAc $(130 \mathrm{~mL})$. The reaction flask was equipped with a mechanical stirrer, nitrogen inlet, and Dean-Stark trap filled with toluene. A stirred oil bath equipped with a thermocouple was used to regulate the reaction temperature. The oil bath temperature was raised to $155{ }^{\circ} \mathrm{C}$ to bring the reaction to reflux and subsequently $\mathrm{K}_{2} \mathrm{CO}_{3}(78.48$ mmol, $10.8467 \mathrm{~g})$ and toluene $(65 \mathrm{~mL})$ were added into the flask. The reaction was azeotropically dehydrated at $155^{\circ} \mathrm{C}$ until water condensation ceased $(\sim 6 \mathrm{~h})$. The temperature was gradually increased to $175^{\circ} \mathrm{C}$, the water-toluene mixture was drained from the Dean-Stark trap and the toluene in the reaction was collected. After $48 \mathrm{~h}$, the reaction mixture was cooled to room temperature. The polymer solution was filtered to remove salt and then was precipitated into isopropyl alcohol as a white solid. The oligomer was filtered, washed with isopropyl alcohol, and dried under vacuum at $120^{\circ} \mathrm{C}$ for $24 \mathrm{~h}$ (yield $>90 \%$ ).

Conversion of amine-endcapped oligomers to acrylamide-endcapped oligomers (AA-BisASXX, Figure 2))

A representative acrylamide functionalization reaction was conducted as follows. Am-BisAS50 (0.517 mmol, $3.000 \mathrm{~g})$ was dissolved in DMAc $(45 \mathrm{~mL})$ in a 100-mL round bottom flask equipped with a magnetic stir bar. The flask was sealed with a septum, purged with nitrogen, and placed in an ice bath. An acid scavenger, TEA (10 mmol, $0.5856 \mathrm{~g})$, was injected into the flask. An excess of acryloyl chloride (3.47 mmol, $0.3143 \mathrm{~g}$ ) dissolved in DMAc (5 mL) was slowly injected into the reaction flask to avoid a rapid exothermic reaction, yielding an opaque solution due to formation of insoluble triethylamine hydrochloride salt. A large excess of acryloyl chloride was used to ensure $100 \%$ endgroup conversion. The reaction was allowed to proceed for $4 \mathrm{~h}$ at $0{ }^{\circ} \mathrm{C}$. Triethylamine hydrochloride salt is soluble in alcohols; the acrylamide-terminated product was precipitated in isopropyl alcohol as a white solid. The product was filtered, washed with isopropyl alcohol to eliminate any residual salt or reaction solvent, and dried in a vacuum oven at $120^{\circ} \mathrm{C}$ for $24 \mathrm{~h}$.

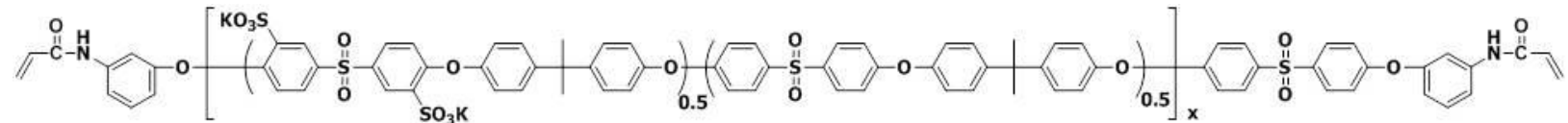

Figure 2. Acrylamide-functional poly(arylene ether sulfone) oligomer with $50 \%$ of the repeat units disulfonated (AA-BisAS50)

Crosslinking and Dense Film Fabrication of Acrylamide-functional Disulfonated Poly(arylene ether sulfone) Oligomers (AA-BisASXX)

AA-BisASXX oligomers were photo-crosslinked in combination with multifunctional acrylates or acrylamides. The crosslinking conditions were optimized via crosslinking experiments with AA-BisAS50, pentaerythritol tetraacrylate (PETA), and 2,2-dimethoxy-2-phenylacetophenone (DMPA) initiator in DMAc solutions. A series of oligomer to PETA molar ratios (1:1, 1:1.5, 1:2 and 1:2.5) and the effect of DMPA concentration on the degree of crosslinking were investigated. Experiments with two, five and ten $\%(\mathrm{wt} / \mathrm{wt})$ initiator relative to total monomer 
mass were conducted. A representative photo-crosslinking reaction is described. AA-BisAS50 glass vial. DMPA (2 wt \%, $0.05 \mathrm{mmol}, 0.012 \mathrm{~g}$ ) was added. The solution was cast on a glass substrate on a level surface and crosslinked by exposure to UV light using a Heraeus LC6B benchtop conveyor instrument $\left(315-400 \mathrm{~nm}\right.$ broad range irradiation, $1.988 \mathrm{~W} / \mathrm{cm}^{2}, 1200 \mathrm{~J} / \mathrm{cm}^{2}$, $15 \mathrm{~s} /$ pass on the conveyor belt) for a total of $150 \mathrm{~s}$. UV curing yielded transparent, free-standing yet fragile polymer films. The films were dried under vacuum at $120{ }^{\circ} \mathrm{C}$ for one day to remove residual solvent. Crosslinking reactions of water soluble AA-BisASXX with 60,80 and $100 \%$ of the repeat units disulfonated were conducted in aqueous solutions using 1-hydroxycyclohexyl phenyl ketone (HCPK) as the photoinitiator and diethylene glycol diacrylate instead of PETA as the crosslinking agent. Similarly, AA-BisASXX (60, 80 and 100) films were crosslinked from methanol-water $(50: 50, \mathrm{v}: \mathrm{v})$ solutions in the presence of one of the multifunctional acrylates: di(ethylene glycol) diacrylate, glycerol propoxylate triacrylate, or trimethylolpropane ethoxylate triacrylate.

\section{Polymer Characterization \\ Nuclear magnetic resonance spectroscopy (NMR)}

${ }^{1} \mathrm{H}$ NMR analysis of the oligomers was performed on a Varian Unity Plus spectrometer and a Varian Inova spectrometer, both operating at $400 \mathrm{MHz}$. The spectra of the oligomers were obtained from a $15 \%(\mathrm{w} / \mathrm{v}) 1-\mathrm{mL}$ solution in $\mathrm{DMSO}-\mathrm{d}_{6}$.

\section{Fourier Transform Infrared Spectroscopy (FT-IR)}

FT-IR experiments were conducted on a Varian 670-IR spectrometer. A background spectrum of a polished $\mathrm{KBr}$ disc was collected before taking sample spectra. Thin films of polymer solutions were coated on the discs and FT-IR spectra were collected in quick succession after UV exposure.

\section{Atomic Force Microscopy (AFM)}

Samples were imaged using a Veeco Multimode AFM. The samples were equilibrated for $12 \mathrm{~h}$ at $30{ }^{\circ} \mathrm{C}$ and $40 \%$ relative humidity and then sealed prior to imaging. Humidity was measured to be between 25 and $40 \%$ during AFM microscopy sessions. A low force constant Nanosensors Point probe-Plus Si tip with a force constant between 1.2-2.9 N/m was used.

Scanning Electron Microscopy (SEM)

A LEO (Zeiss) 1550 field-emission SEM was used to conduct FE-SEM microscopy. Prior to FESEM experiments, the nonwoven backing of the thin film composites was manually removed. The remaining layers which included a dense UV-crosslinked top layer and a UDEL ${ }^{\circledR}$ foam support were freeze-fractured using liquid nitrogen. Freeze-fractured samples were sputter coated with 10-12 nm of a gold-palladium alloy immediately before SEM imaging.

\section{Water Uptake}


Water uptake of crosslinked films was measured gravimetrically. Films were dried at $120{ }^{\circ} \mathrm{C}$ under vacuum overnight to obtain the dry weight $\left(W_{d r y}\right)$. The films were then immersed in DI water at ambient temperature for at least $48 \mathrm{~h}$. The films were removed, quickly blotted to remove water droplets on the surface, and weighed to obtain the wet weight $\left(W_{\text {wet }}\right)$. Equation 1 shows the calculation used to determine the gravimetric percent water uptake.

$$
\text { Water Uptake }(\%)=\frac{\mathrm{W}_{\text {wet }}-\mathrm{W}_{\text {dry }}}{\mathrm{W}_{\text {dry }}} \times 100
$$

\section{Equation 1}

\section{Gel fractions}

Gel fraction measurements were performed to determine the extent of crosslinking. Crosslinked films were dried at $120^{\circ} \mathrm{C}$ under vacuum overnight. Then $\sim 0.2 \mathrm{~g}$ of initial sample was placed in a 20-mL scintillation vial filled with DMAc and stirred at $100{ }^{\circ} \mathrm{C}$ overnight. The remaining solid was filtered, transferred to a pre-weighed vial, and dried at $120{ }^{\circ} \mathrm{C}$ under vacuum overnight. The final weight was taken the next day. At least three samples were extracted and the gel fractions were averaged. Gel fractions were calculated using equation 2.

$$
\text { Gel Fraction }(\%)=\frac{W_{\text {final }}}{W_{\text {initial }}} \times 100
$$

\section{Results and Discussion}

\section{Oligomer Synthesis}

Direct copolymerization of partially disulfonated poly(arylene ether sulfones) (Figure 1) synthesized via nucleophilic aromatic substitution step polymerizations have been previously described by McGrath et al. ${ }^{13,17,23,26-29}$ The 3,3'-disulfonated-4,4'-dichlorodiphenylsulfone (SDCDPS) monomer was used to control the degree of sulfonation and therefore the hydrophilicity of the copolymers. ${ }^{13,14}$ Oligomers with targeted molecular weights of $\sim 5,000$ $\mathrm{g} / \mathrm{mol}$ were investigated in this research utilizing a monofunctional endcapping reagent to limit the molecular weights. A modified Carothers equation was used to calculate the required amount of $m$-aminophenol endcapping agent which also provided aromatic amine endgroup functionality. ${ }^{30} m$-Aminophenol was preferred over $p$-aminophenol because $m$-aminophenol produces more oxidatively stable oligomers. ${ }^{30,31}$ Thus, the $m$-aminophenol endgroup greatly extends the shelf life of these oligomers. ${ }^{31}$ Toluene was used as the azeotropic solvent to remove the byproduct water that the $\mathrm{K}_{2} \mathrm{CO}_{3}$ reaction produces. The toluene reflux was continued until no more water was observed in the distillate. The temperature was raised slowly to $175{ }^{\circ} \mathrm{C}$, and toluene was slowly collected until the initial charged volume was obtained. The viscosity of the 
reaction solution increased slightly after 48 hours. Since the targeted molecular weight was 5,000 $\mathrm{g} / \mathrm{mol}$, no drastic change in viscosity was anticipated. The nucleophilic substitution reaction between amine-terminated oligomers and acryloyl chloride occurs rapidly. Therefore the reaction was cooled to $0{ }^{\circ} \mathrm{C}$ before adding triethylamine and acryloyl chloride. The am-BisAS50 was dried prior to reaction with the acid chloride to avoid reaction between water and the acryloyl chloride.

\section{Oligomer Characterization}

The composition of the amino-functional oligomer was characterized by ${ }^{1} \mathrm{H}$ NMR to determine the degree of sulfonation (D.S.) and to estimate the $M_{n}$. A ${ }^{1} \mathrm{H}$ NMR spectrum of the AMBisAS50 oligomer is shown in Figure 3. The spectrum did not show any side products, residual monomer or reaction solvent. The D.S. was calculated using equation 3. The measured D.S. was $49 \%$ compared to the targeted D.S. of $50 \%$.

$$
\text { D.S. }(\%)=\frac{\left[\left(\mathrm{H}_{a}+\mathrm{H}_{b}+\mathrm{H}_{c}\right) / 3\right] / 2}{\left[\left(\mathrm{H}_{a}+\mathrm{H}_{b}+\mathrm{H}_{c}\right) / 3\right] / 2+\left[\left(\mathrm{H}_{f}+\mathrm{H}_{g}\right) / 2\right] / 4} \times 100
$$

\section{Equation 3}

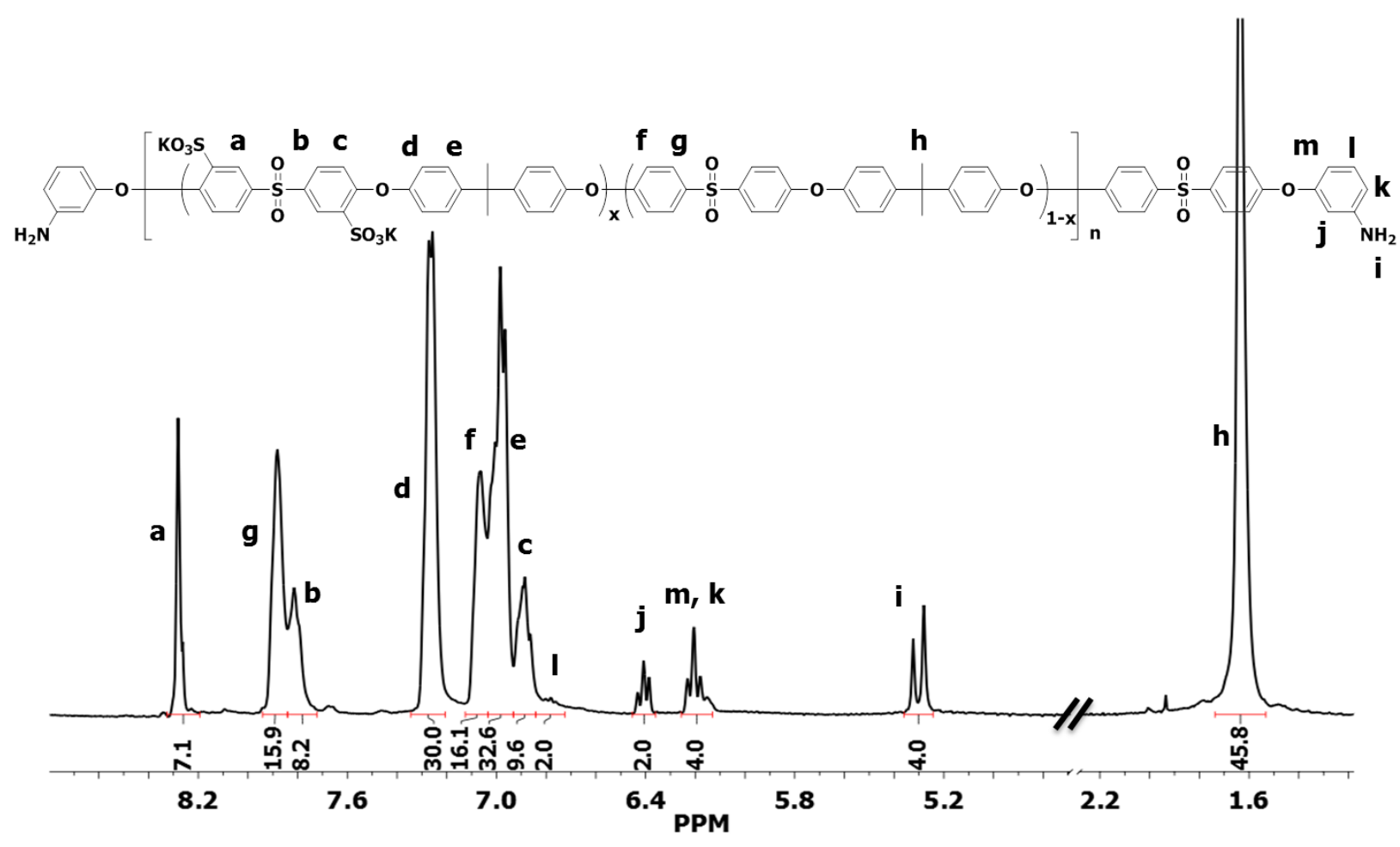

Figure 3. ${ }^{1} \mathrm{H}$ NMR of am-BisAS50 
The $m$-aminophenol endgroups can be attached to a non-sulfonated or a disulfonated unit so the positions of the $m$-aminophenol protons shift slightly depending on which monomer is the adjacent group. For example, the singlets (i) are due to amine protons bonded to DCDPS (further downfield) and SDCDPS. Similarly, endgroup peaks $(\mathbf{j}),(\mathbf{m})$, and (k) have overlapping, slightly shifted signals depending on the adjacent group.

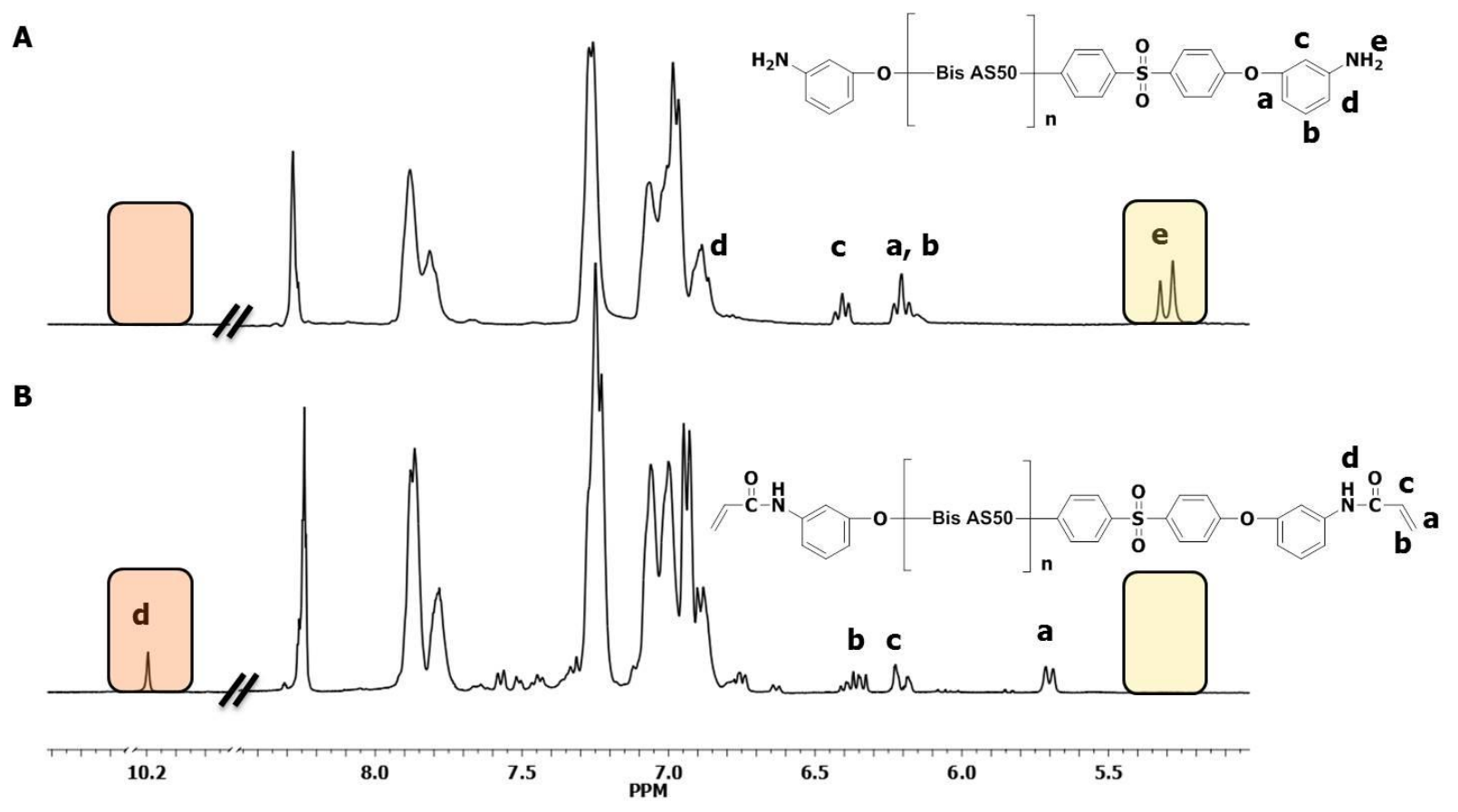

Figure 4. The endgroups of oligomers were modified as evidenced by ${ }^{1} \mathrm{H}$ NMR spectra. A: ${ }^{1} \mathrm{H}$ NMR of am-BisAS50, and B: ${ }^{1} \mathrm{H}$ NMR of AA-BisAS50

${ }^{1} \mathrm{H}$ NMR was also utilized to confirm the endgroup structures of the acrylamide-functional oligomers (Figure 4). As expected, the amine peak disappeared upon conversion to the acrylamide while the amide proton at approximately $10.2 \mathrm{ppm}$ appeared. The $M_{n}$ calculated by ${ }^{1} \mathrm{H}$ NMR endgroup analysis was $4,900 \mathrm{~g} / \mathrm{mol}$, as determined by the ratio of the integral values of the aromatic protons of DCDPS (at $7.89 \mathrm{ppm}$ ) and SDCDPS (7.79 ppm and $8.26 \mathrm{ppm}$ ) that are adjacent to sulfonyl groups and the acrylamide endgroup peak (doublet at 5.72 and $5.74 \mathrm{ppm}$ ).

\section{Crosslinking and Film Fabrication of AA-BisASXX Oligomers}

The acrylamide-functional oligomers were photochemically crosslinked using 2,2-dimethoxy-2phenylacetophenone (DMPA) or 1-hydroxycyclohexyl phenyl ketone (HCPK) as the photoinitiator in the presence of multifunctional acrylates. The efficiencies of these photoinitiators and the mechanisms involved in their crosslinking reactions with acrylates and acrylamides have been extensively studied. ${ }^{32}$ The crosslinking yielded transparent and fragile polymer films. Reactions of the acrylamide-functional polysulfone copolymer in the absence of a 
multifunctional acrylate co-monomer, however, only produced networks with gel fractions ranging from 13 to $45 \%$. Addition of a highly functional crosslinking agent increases the concentration of reactive groups and drives the crosslinking reactions to high gel fractions.

FT-IR experiments were conducted to observe the photo-crosslinking of these acrylamideterminated oligomers with the multifunctional acrylates. FT-IR is a powerful tool for characterizing crosslinked networks because infrared spectroscopy is sensitive to many types of functional groups and insoluble networks can be characterized directly. The bending $=\mathrm{C}-\mathrm{H}$ vibrational mode of the acrylate endgroups shows a characteristic peak at $812 \mathrm{~cm}^{-1}$. ${ }^{43,44}$ The FTIR spectrum (1 in Figure 5) of an initial mixture of BISAS50, PETA and DMPA shows a peak at $812 \mathrm{~cm}^{-1}$, indicating the presence of the double bonds prior to photo-crosslinking. ${ }^{33,34}$ Upon exposure to UV light the absorbance at $812 \mathrm{~cm}^{-1}$ disappears, showing the consumption of unsaturated endgroups. Ultrathin films were coated on polished $\mathrm{KBr}$ discs for the FT-IR experiments. The spectra showed that these ultrathin films were completely crosslinked after 30 seconds of exposure to UV irradiation. Table 1 summarizes the gel fractions of dense membranes with the same composition that were in the range of 40-50 $\mu \mathrm{m}$ thick. Gel fraction analysis indicated that those membranes required up to $150 \mathrm{~s}$ of UV irradiation to form a crosslinked network with both reactants incorporated almost quantitatively.

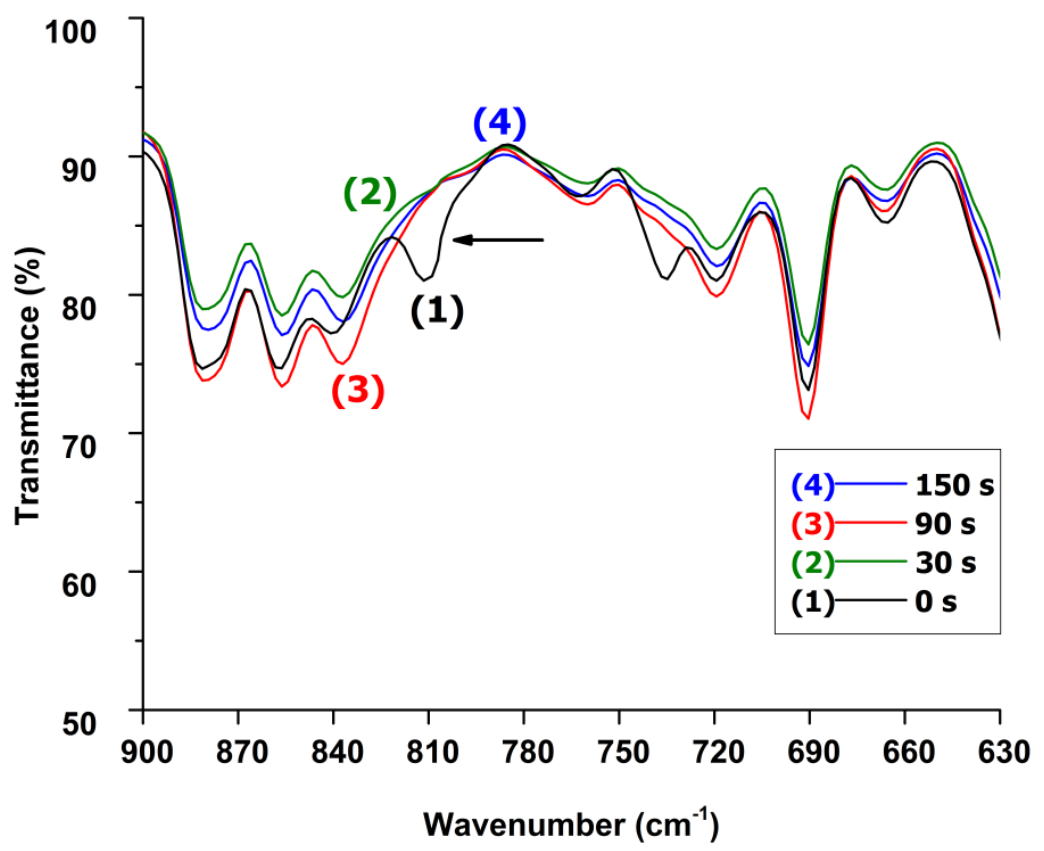

Figure 5. FT-IR spectra of a reaction mixture of BisAS50 $(0.1 \mathrm{mmol})$, PETA $(0.25 \mathrm{mmol})$ and DMPA $(2$ wt $\%)$ before $(1)$ and after $(2,3,4)$ photo-crosslinking

\section{Crosslinking Agent and Photoinitiator Concentrations}

The role of the ratio of AA-BisAS50 to PETA was investigated to assess the influence of the acrylate crosslinking agent concentration on the degree of crosslinking of dense films with a thickness of $\sim 150 \mu \mathrm{m}$. The AA-BisAS50 to PETA molar ratio ranged from 1.0:1.0 to 1.0:2.5 with $2 \mathrm{wt} \%$ of DMPA. Gel fractions of up to $87 \%$ were obtained. The gel fraction increased 
linearly and plateaued around $87 \%$ with increasing PETA concentration. This systematic study indicated that at least a 1.0 to 2.0 molar ratio of AA-BisAS50 to PETA is required to achieve a high degree of crosslinking with these oligomers. Experiments to investigate the effect of photoinitiator concentration on the degree of crosslinking were also conducted with a molar ratio of oligomer to PETA of 1.0:2.5. Initiator concentration was varied from 2, 5 and $10 \mathrm{wt} \%(0.05$ to 0.5 mmoles of initiator relative to $1.2 \mathrm{eq}$ of double bonds) relative to the total amount of AABisAS50 and PETA. Increasing the initiator concentration did not yield a significant increase in the crosslink density. In fact, gel fractions decreased at higher initiator concentrations and this was attributed to radical recombination as a result of increasing radical concentration. ${ }^{32}$

\section{The Effect of Film Thickness on Degree of Crosslinking}

The Beer-Lambert law ${ }^{35,36}$ states that absorbance correlates with molar absorptivity, concentration and thickness of the light absorbing medium. Therefore, high thickness of a dense film may have an adverse effect on the total degree of crosslinking. To investigate this aspect, dense films of two thicknesses were investigated. Thick $150-\mu \mathrm{m}$ films were cast directly from solution onto a glass substrate and $50-\mu \mathrm{m}$ films were cast with the aid of a doctor blade. Gel fractions of these networks were not significantly different. This indicates that the UV absorption and photo-polymerization had occurred throughout the thickness range investigated.

\section{The Influence of UV Exposure on Crosslinking of AA-BisAS50-PETA Films}

$\mathrm{UDEL}^{\circledR}$ poly(arylene ether sulfone)s absorb UV radiation between wavelengths of $\sim 230$ and 310 $\mathrm{nm}$ with a maximum around $270 \mathrm{~nm} .{ }^{37}$ Photodegradation, chain scission and crosslinking can occur in a broad temperature window ranging from ambient temperature to $250{ }^{\circ} \mathrm{C}$. The extent of these photochemical reactions depends on temperature and exposure time. ${ }^{37,38}$ Extended exposure to UV radiation at elevated temperatures photochemically degrades such poly(arylene ether sulfone)s. Thus, photo-crosslinking of AA-BisASXX copolymers was conducted utilizing a broad range of UV radiation $(315-400 \mathrm{~nm})$ outside the absorbance window of these copolymers. Additionally, in this work, the materials were only exposed to UV radiation for a maximum of $150 \mathrm{~s}$ and at $\sim 75^{\circ} \mathrm{C}$. Therefore significant photodegradation was not expected due to the mild crosslinking conditions.

$$
\mathrm{R}_{\mathrm{p}}=\Phi_{\mathrm{m}} \times \mathrm{I}_{\mathrm{abs}}
$$

Equation 4

$$
\mathrm{Ri}=\Phi \mathrm{i} \times \mathrm{I}_{\mathrm{abs}}
$$

Equation 5

In the course of optimizing the reaction conditions, the effect of the length of UV exposure was studied. Duration of exposure to UV irradiation influenced the gel fractions of these networks (Table 2). Increasing the UV exposure time from 30 to 150 seconds resulted in up to a $50 \%$ increase in gel fraction. We attribute this increase to the amount of energy absorbed during photo-crosslinking. The rate of photopolymerization $\left(\mathrm{R}_{\mathrm{p}}\right)$ depends on the polymerization quantum yield $\left(\Phi_{\mathrm{m}}\right)$ which defines the number of double bonds polymerized per photon absorbed and the amount of absorbed energy at a specific wavelength $\left(\mathrm{I}_{\mathrm{abs}}\right)$. Equation 4 defines this 
relationship. Similarly in Equation 5, where $R_{i}$ is the rate of initiation, the rate of initiation depends on the initiation quantum yield $\Phi_{i}$ which describes the number of initiated chains per photon absorbed and $\mathrm{I}_{\mathrm{abs}}{ }^{32}$ Therefore prolonging the UV exposure increases the amount of energy absorbed $\mathrm{I}_{\mathrm{abs}}$ which increases $\mathrm{R}_{\mathrm{p}}$ and $\mathrm{R}_{\mathrm{i}}$ of the photopolymerizations.

Table 2. Gel fractions of crosslinked films $\sim 150 \mu \mathrm{m}$ thick with varied reactant molar ratios and durations of UV exposure with $2 \mathrm{wt} \%$ DMPA photoinitiator

\begin{tabular}{|c|c|c|c|}
\hline \multirow{2}{*}{$\begin{array}{c}\text { AA-BISAS50: PETA } \\
\text { Molar Ratio }\end{array}$} & \multicolumn{3}{|c|}{ \% Gel Fraction after Photo-crosslinking } \\
\cline { 2 - 4 } & 30 seconds & 90 seconds & 150 seconds \\
\hline $1.0: 1.0$ & $29 \pm 2$ & $63 \pm 2$ & $82 \pm 0.1$ \\
\hline $1.0: 1.5$ & $30 \pm 1$ & $70 \pm 1$ & $85 \pm 0.1$ \\
\hline $1.0: 2.0$ & $37 \pm 1$ & $74 \pm 2$ & $87 \pm 0.3$ \\
\hline $1.0: 2.5$ & $43 \pm 1$ & $75 \pm 2$ & $87 \pm 0.4$ \\
\hline
\end{tabular}

The presence of the photoinitiator is crucial for achieving a high degree of crosslinking. Experiments without a photoinitiator failed to produce crosslinked films. Thus this method requires a photoinitiator that is soluble in the casting solvent. DMPA is a water-insoluble photoinitiator, whereas HCPK is water-soluble. Experiments were conducted to compare networks produced with HCPK or DMPA in DMAc solutions. Networks initiated with HCPK and with an oligomer to PETA or diethylene glycol diacrylate ratio of 1:2.5 produced gel fractions of 80 and $70 \%$ respectively (slightly lower than the corresponding network from PETA/DMPA). This result may be attributed to the lower functionality of diethylene glycol diacrylate $(\mathrm{f}=4)$ relative to PETA $(\mathrm{f}=8)$. Differences in results with the two photoinitiators may be at least partially attributed to the different quantum efficiency of each under these experimental conditions.

\section{Preparation of UV Crosslinked AA-BisASXX from Benign Solutions}

In order to obtain very thin film selective layers, ${ }^{29}$ TFCs prepared from water or water/alcohol solutions were investigated. Solutions containing AA-BisASXX, an acrylate crosslinker and 2 wt\% of the HCPK photoinitiator were cast on a commercial UDEL ${ }^{\circledR}$ film support and UVcrosslinked to form TFCs. DMAc, as well as other organic solvents such as DMF, DMSO, and NMP are good solvents for disulfonated oligomers, the acrylate crosslinking reagents and the photoinitiators. For example casting solutions of BisAS50, PETA and DMPA in DMAc that have been discussed thus far also dissolve the UDEL ${ }^{\circledR}$ film support and this excludes them as potential casting solvents. ${ }^{22}$

\section{Aqueous Casting Solution}

AA-BisASXX (XX = 60, 80 or 100), diethylene glycol diacrylate and the HCPK photoinitiator were dissolved in water and thin films were cast on the UDEL ${ }^{\circledR}$ film support to form TFCs. 
Water is a non-solvent for $\mathrm{UDEL}^{\circledR}$ polysulfone, and thus, its use for casting preserves the polysulfone support film structure. This is crucial to prevent pore plugging, and densification or collapse due to swelling. Avoiding these undesirable outcomes is critical to obtaining thin films suitable for the measurement of transport properties. While the disulfonated polysulfone oligomers with $50 \%$ of the units disulfonated are water-insoluble, AA-BisASXX (XX $=60,80$ or 100) are water-soluble. Replacing the water-insoluble PETA with soluble diethylene glycol diacrylate and photo-crosslinking of the water-soluble oligomers yielded networks with gel fractions of $\sim 75 \%$ from aqueous solutions. This photo-crosslinking procedure conducted in aqueous solutions of these disulfonated poly(arylene ether sulfone)s is a novel method to the best of our knowledge.

\section{Methanol:Water (50:50, v:v) Casting Solution}

In addition to water, other polar solvents such as alcohols are also non-solvents for UDEL ${ }^{\circledR}$ polysulfone and good solvents for the AA-BisASXX oligomers with high degrees of sulfonation. Like diethylene glycol diacrylate, glycerol propoxylate triacrylate and trimethylolpropane ethoxylate triacrylate are multifunctional acrylates $(\mathrm{f}=6)$ that are soluble in polar solvents such as methanol or methanol-water mixtures. Glycerol propoxylate triacrylate and trimethylolpropane ethoxylate triacrylate have higher functionality than diethylene glycol diacrylate $(f=6 \mathrm{vs} f=4)$ and thus, it was expected that they would yield networks with a higher degree of crosslinking. AABisAS60 150 micron thick film samples were crosslinked from water:methanol (50:50, v:v) solutions in the presence of HCPK. As anticipated, the acrylate crosslinking agents with the higher functionalities yielded films with gel fractions above $90 \%$. Table 3 summarizes the results of gel fraction analysis of AA-BisAS60 films crosslinked with a molar ratio of the oligomer to the multifunctional acrylate crosslinking agent of 1:2.5. Both aqueous and water-methanol solutions produced high gel fractions that enabled successful fabrication of thin films from benign solvents.

Table 3. Gel fractions of networks containing AA-BISAS60 crosslinked with multifunctional acrylates in the presence of $2 \mathrm{wt} \%$ of $\mathrm{HCPK}$ as the photoinitiator, cast from 50:50 v:v methanol:water.

463

\section{4}

\begin{tabular}{|c|c|c|}
\hline Acrylate Crosslinking Agent & Functionality & $\%$ Gel Fraction \\
\hline Diethylene glycol diacrylate & 4 & $83 \pm 5$ \\
\hline Trimethylolpropane ethoxylate triacrylate & 6 & $94 \pm 5$ \\
\hline Glycerol propoxylate triacrylate & 6 & $98 \pm 1$ \\
\hline
\end{tabular}

465

466

467

Water Uptake 
Water uptake of photocrosslinked films of BisASXX (XX=60-100) copolymerized with glycerol propoxylate triacrylate with $2 \mathrm{wt} \%$ HCPK in 50:50 v:v methanol:water were measured. The degree of sulfonation correlates with water uptake of these networks, where films containing BisAS60, BisAS80 and BisAS100 absorbed 49, 90 and 143 weight \% respectively. These films swelled due to their high ionic content but the crosslinks limited the degree of swelling. It is hypothesized that a selective layer with high water uptake cast on UDEL ${ }^{\circledR}$ film would result in a thin film composite with significantly higher flux compared to a conventional thin film composite prepared with an interfacially-crosslinked polyamide layer.

Surface Morphology of Photo-Crosslinked Thin Films by Atomic Force Microscopy (AFM) and Scanning Electron Microscopy (SEM)
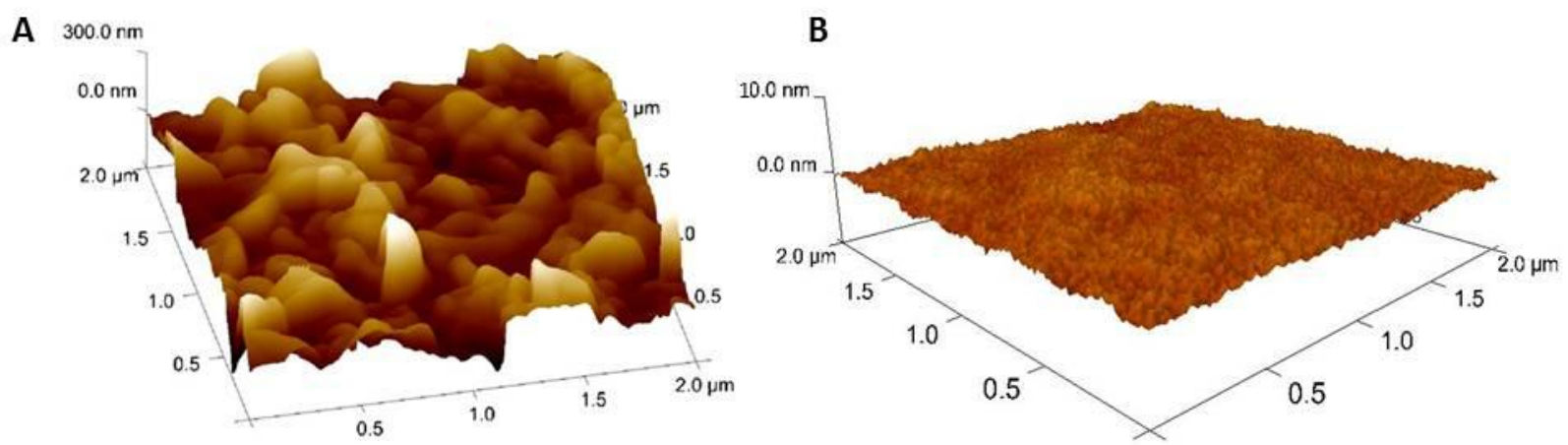

Figure 6. AFM micrographs of (A) a polyamide TFC, ${ }^{22}$ and (B) an AA-BisAS60 TFC

State-of-the-art polyamide membranes display a very different surface topology compared to BisASXX thin films due to differing methods of fabrication. The polyamide thin film membrane surface retains a very rough surface texture, likely as a result of the interfacial polymerization and crosslinking reaction of aromatic tri-functional acid chlorides and aromatic diamines that takes place as the thin film is formed. ${ }^{39,40}$ Root-mean-square roughness (RMS) can be used to quantify the surface topology. RMS roughness is a measure of the standard deviation of the surface heights with smooth surfaces having small RMS values. Figure 6 (A) shows an AFM micrograph of a polyamide thin film membrane with a separation layer that was prepared by interfacial polymerization. ${ }^{22}$ Fouling has been attributed to the extremely rough surface structure of the polyamide membranes. The rough surface significantly diminishes the water flux of the membranes over time. ${ }^{41-43}$ The AFM micrograph of a BisAS60 film in Figure 6 (B) shows a smooth surface that is confirmed by the RMS value. Solvent cast films of BisASXX had RMS surface roughness values between $0.4-0.7 \mathrm{~nm}$ whereas the commercial polyamide had a value of $56.2 \mathrm{~nm}$ as described previously. ${ }^{22}$ It is hypothesized that the smooth surface is primarily due to film formation prior to crosslinking. The smooth surface of the photo-crosslinked membrane coupled with the hydrophilic surface is expected to resist fouling and maintain water flux over a longer service life. 
The thin selective layer of these composite films on the UDEL $₫$ support was confirmed with cross-sectional SEM micrographs (Figure 7). The skin surface illustrates the absence of "ridges and valleys" that are inherent to the interfacially polymerized polyamide films. The images show a selective dense top layer with a relatively uniform thickness of approximately $600 \mathrm{~nm}$ and a smooth surface.
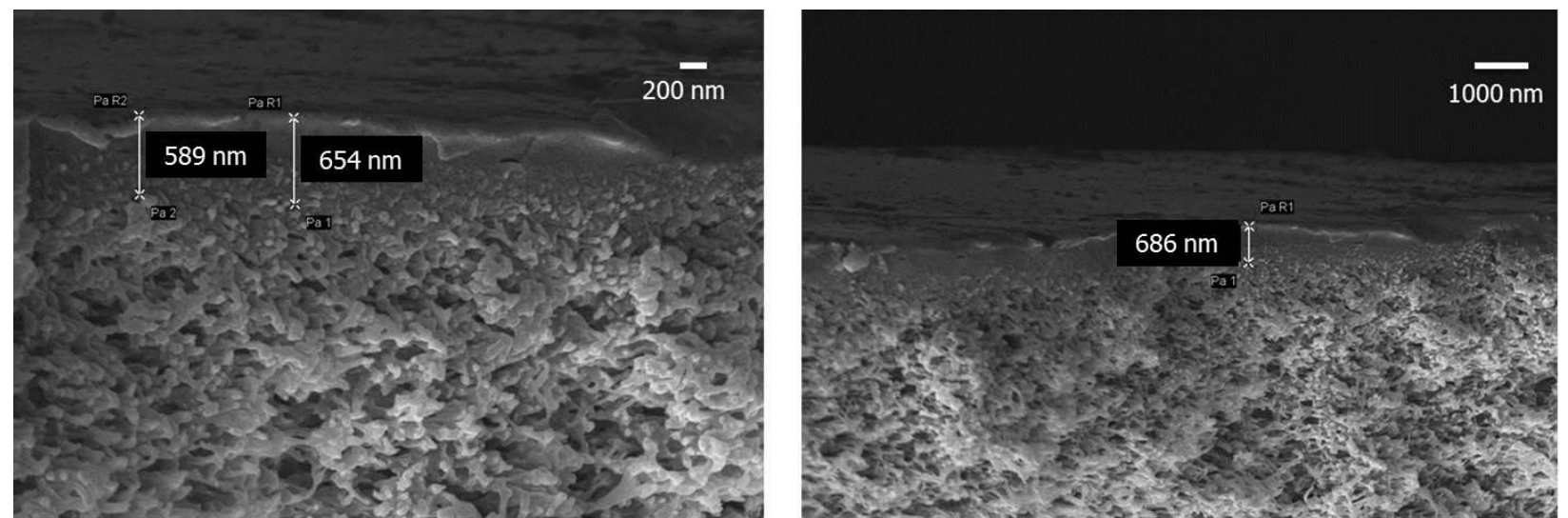

Figure 7. SEM cross-sectional micrographs of a photo crosslinked films containing AA-BisAS60 and glycerol propoxylate triacrylate

\section{Conclusions}

Our research has focused on the synthesis of novel UV crosslinkable oligomers and potential methods to prepare thin films utilizing an aqueous-based UV curing procedure. Novel UV crosslinkable oligomers were synthesized for potential applications such as water purification membranes. The UV crosslinking reaction conditions, for instance, the molar ratio of reagents, type and concentration of crosslinking agent with two photoinitiators were evaluated. For the first time, UV crosslinking of partially or fully disulfonated poly(arylene ether sulfone) oligomers with acrylamide endgroups was demonstrated from methanol-water and aqueous solutions, thereby enabling application onto a commercial polysulfone foam support structure. UV crosslinking produced films with up to $98 \%$ gel fractions. Early results on fabrication of well-defined thin films were demonstrated utilizing a rapid aqueous based casting/crosslinking procedure under UV radiation. AFM micrographs demonstrated that the photocured films exhibited two orders of magnitude lower RMS values compared to interfacially polymerized polyamide films. SEM micrographs corroborate the AFM measurements. Moreover, SEM crosssectional micrographs showed the presence of a film layer with a thickness of $\sim 600 \mathrm{~nm}$.

\section{Acknowledgements}

The authors are grateful for the support of Dow Water \& Process Solutions, Inc. This research was also supported by the U.S. National Science Foundation Partnerships for InnovationAccelerating Innovative Research (PFI-AIR, Grant \#1237858) and Partnerships for Innovation (PFI) -Partnerships for Water Purification (Grant \#0650277). 


\section{References:}

1. T. Nishikubo, A. Kameyama, H. Kudo, In Photoinitiated Polymerization; American Chemical Society, 2003, pp 363-377.

2. R. Schwalm, In Polymer Science: A Comprehensive Reference; Möller, K. M., Ed.; Elsevier: Amsterdam, 2012, pp 567-579.

3. C. L. Henderson, In Polymer Science: A Comprehensive Reference; Elsevier: Amsterdam, 2012, pp 3776.

4. C. G. Roffey. Photopolymerization of surface coatings; Wiley: Chichester ;New York, 1982.

5. D. J. Lipomi, R. V. Martinez, L. Cademartiri, G. M. Whitesides, In Polymer Science: A Comprehensive Reference; Elsevier: Amsterdam, 2012, pp 211-231.

6. J.-P. Fouassier. Munich; New York; Cincinnati, 1995.

7. Z. W. Wicks, In Encyclopedia of Polymer Science and Technology; John Wiley \& Sons, Inc., 2002.

8. Z. W. Wicks, In Encyclopedia of Polymer Science and Technology; John Wiley \& Sons, Inc., 2002.

9. J. B. Rose. Polymer 1974, 15, 456-465.

10. B. E. Jennings, M. E. B. Jones, J. B. Rose. J. Polym. Sci., Polym. Symp. 1967, 16, 715-724.

11. R. N. Johnson, A. G. Farnham, R. A. Clendinning, W. F. Hale, C. N. Merriam. Journal of Polymer Science Part A-1: Polymer Chemistry 1967, 5, 2375-2398.

12. L. M. Robeson, A. G. Farnham, J. E. McGrath. Midl. Macromol. Monogr. 1978, 4, 405-425.

13. F. Wang, M. Hickner, Y. S. Kim, T. A. Zawodzinski, J. E. McGrath. J. Membr. Sci. 2002, 197, 231-242.

14. F. Wang, Y. Kim, M. Hickner, T. A. Zawodzinski, J. E. McGrath. Polym. Mater. Sci. Eng. 2001, 85, 517518.

15. X. Wei, P. Ho-Bum, J. Cook, L. Chang Hyun, B. Gwangsu, B. D. Freeman, J. E. McGrath. Water Science \& Technology 2010, 61, 619-624.

16. G. M. Geise, H. B. Park, A. C. Sagle, B. D. Freeman, J. E. McGrath. Journal of Membrane Science 2011, 369, 130-138.

17. M. Paul, H. B. Park, B. D. Freeman, A. Roy, J. E. McGrath, J. S. Riffle. Polymer 2008, 49, 2243-2252.

18. R. F. Service. Science 2006, 313, 1088-1090.

19. J. E. Cadotte, R. J. Petersen, R. E. Larson, E. E. Erickson. Desalination 1980, 32, 25-31.

20. A. Noshay, L. M. Robeson. J. Appl. Polym. Sci. 1976, 20, 1885-1903.

21. B. C. Johnson, I. Yilgor, C. Tran, M. Iqbal, J. P. Wightman, D. R. Lloyd, J. E. McGrath. J. Polym. Sci., Polym. Chem. Ed. 1984, 22, 721-737.

22. C. H. Lee, B. D. McCloskey, J. Cook, O. Lane, W. Xie, B. D. Freeman, Y. M. Lee, J. E. McGrath. Journal of Membrane Science 2012, 389, 363-371.

23. B. J. Sundell, K.-s. Lee, A. Nebipasagil, A. Shaver, J. R. Cook, E.-S. Jang, B. D. Freeman, J. E. McGrath. Ind. Eng. Chem. Res. 2014, 53, 2583-2593.

24. M. Sankir, V. A. Bhanu, W. L. Harrison, H. Ghassemi, K. B. Wiles, T. E. Glass, A. E. Brink, M. H. Brink, J. E. McGrath. J. Appl. Polym. Sci. 2006, 100, 4595-4602.

25. Y. Li, R. A. VanHouten, A. E. Brink, J. E. McGrath. Polymer 2008, 49, 3014-3019.

26. Y. S. Kim, M. A. Hickner, L. Dong, B. S. Pivovar, J. E. McGrath. J. Membr. Sci. 2004, 243, 317-326.

27. W. L. Harrison, M. A. Hickner, Y. S. Kim, J. E. McGrath. Fuel Cells (Weinheim, Ger.) 2005, 5, 201-212.

28. H. B. Park, B. D. Freeman, Z.-B. Zhang, G.-Y. Fan, M. Sankir, J. E. McGrath. PMSE Prepr. 2006, 95, 889891.

29. W. Xie, J. Cook, H. B. Park, B. D. Freeman, C. H. Lee, J. E. McGrath. Polymer 2011, 52, 2032-2043.

30. M. J. Jurek, J. E. McGrath. Polymer 1989, 30, 1552-1557.

31. G. D. Lyle, J. S. Senger, D. H. Chen, S. Kilic, S. D. Wu, D. K. Mohanty, J. E. McGrath. Polymer 1989, 30, 978-985.

32. J. P. Fouassier, J. Lalevée. Photoinitiators for Polymer Synthesis; Wiley: Somerset, NJ, USA, 2012.

33. C. Decker, F. Masson, R. Schwalm. Macromolecular Materials and Engineering 2003, 288, 17-28. 
34. S. Oprea, S. Vlad, A. Stanciu, M. Macoveanu. European Polymer Journal 2000, 36, 373-378.

584 35. M. Guvendiren, B. Purcell, J. A. Burdick, In Polymer Science: A Comprehensive Reference;

585 Matyjaszewski, K., Möller, M., Eds.; Elsevier: Amsterdam, 2012, pp 413-438.

586 36. R. E. Krebs. Encyclopedia of scientific principles, laws, and theories; Greenwood Press: Westport, 587 Conn., 2008.

588 37. S. Kuroda, A. Nagura, K. Horie, I. Mita. Eur. Polym. J. 1989, 25, 621-627.

589 38. T. Yamashita, H. Tomitaka, T. Kudo, K. Horie, I. Mita. Polym. Degrad. Stab. 1993, 39, 47-54.

590 39. W. E. Mickols, In Polymer Science: A Comprehensive Reference; Matyjaszewski, K., Möller, M., Eds.; 591 Elsevier: Amsterdam, 2012, pp 831-848.

592 40. W. Xie, G. M. Geise, B. D. Freeman, H.-S. Lee, G. Byun, J. E. McGrath. J. Membr. Sci. 2012, 403-404, 593 152-161.

594 41. M. Elimelech, X. Zhu, A. E. Childress, S. Hong. J. Membr. Sci. 1997, 127, 101-109.

595 42. S. Lee, J. Cho, M. Elimelech. Desalination 2004, 160, 1-12.

596 43. E. M. Vrijenhoek, S. Hong, M. Elimelech. Journal of Membrane Science 2001, 188, 115-128.

597

598 

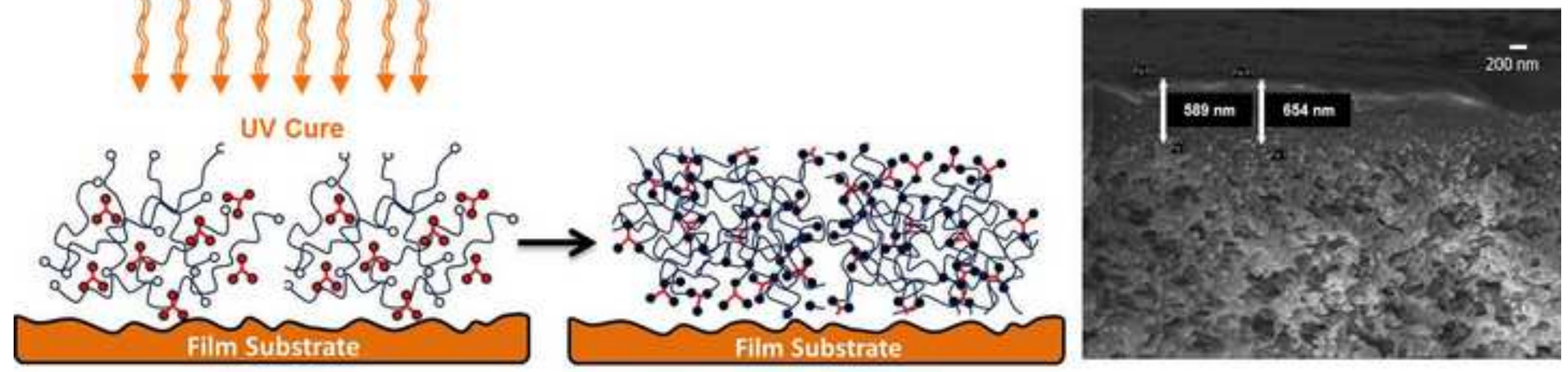